\title{
Maximum entropy with fluctuating constraints The example of K-distributions
}

\author{
J.-F. Bercher ${ }^{1}$ \\ Laboratoire des Signaux et Systèmes, CNRS-Univ Paris Sud-Supelec, 91192 Gif-sur-Yvette cedex, France \\ and Université Paris-Est, LabInfo-IGM, 5 bd Descartes, 77454 Marne la Vallée Cedex 2, France
}

\begin{abstract}
We indicate that in a maximum entropy setting, the thermodynamic $\beta$ and the observation contraint are linked, so that fluctuations of the latter imposes fluctuations of the former. This gives an alternate viewpoint to 'superstatistics'. While a Gamma model for fluctuations of the $\beta$ parameter gives the so-called Tsallis distributions, we work out the case of a Gamma model for fluctuations of the observable, and show that this leads to K-distributions. We draw attention to the fact that these heavy-tailed distributions have high interest in physical applications, and we discuss them in some details.
\end{abstract}

Key words:

maximum entropy principle, superstatistics, K-distributions

PACS: 05.40.-a, 02.50.-r, 05.90.+m

\section{Introduction}

The technique of compounding, or mixing, is a standard technique for the interpretation or construction of distributions [1, chapter 8]. A random variable may result of the mixing of different populations, or the source of the variable may be unknown and the possible sources characterized by a probability density function. This technique has been employed in $[2,3]$ for recovering the Tsallis (Generalized Pareto) distribution and generalized as 'Superstatistics'. This concept that has met interest $[4,5,6,7,8,9]$ consists in varying the natural parameter $\beta$ of a Boltzmann distribution according to some density $f_{\beta}(\beta)$ for $\beta$. In particular, the Tsallis distribution is associated with a Gamma distribution. Interestingly, this distribution had already been derived in such a way in [10].

The objective of this Letter is twofold. First, we relate fluctuations of the intensive parameter $\beta$ to fluctuations of an observable $\bar{E}$, and indicate that the relevant distribution of the underlying system can be viewed as the maximum entropy solution with randomized constraints. Second, we work out the case of a Gamma distribution for the observable and draw attention to the physically relevant solution, a K-distribution, that is characterized and discussed.

Email address: jf .bercher@esiee.fr(J.-F. Bercher).

1 On sabbatical leave from ESIEE-Paris, France. 


\section{Maximum entropy with fluctuating constraints}

Consider a Gibbs ensemble where each system of the ensemble is composed of equilibrated subsystems, cells, each of them being described by the standard canonical factor $e^{-\beta E}$. The corresponding distribution can be regarded as the distribution that maximizes the Shannon-Boltzmann entropy

$$
S=-\int f_{E}(x) \log f_{E}(x) d \Gamma
$$

subject to normalization and to the observation constraint $\bar{E}=\int x f_{E}(x) d \Gamma$, and where $d \Gamma$ is the infinitesimal volume in the phase space. Resolution leads to $f_{E}(x \mid \bar{E})=e^{-\beta x-\log Z(\beta)}$, where $Z(\beta)=\int e^{-\beta E} d \Gamma$ is the partition function and where $\beta$, the Lagrange multiplier associated to the observation constraint is solution of

$$
S(\bar{E})=\max _{\beta}\{-\beta \bar{E}-\log Z(\beta)\} .
$$

This relation simply indicates that the entropy $S(\bar{E})$ and the potential $\Phi(\beta)=\log Z(\beta)$ are conjugated functionals while $\bar{E}$ and $\beta$ are conjugated variables. This also gives the standard relationship

$$
\bar{E}=-\frac{d \Phi(\beta)}{d \beta}=-\frac{d \log Z(\beta)}{d \beta} .
$$

Hence, it is stressed that these variables are not independent, and that variations of the thermodynamic $\beta$ necessarily implies variations of its dual variable $\bar{E}$ and reciprocally. Thus, if the mean energy varies from subsystem to subsystem (from cell to cell), and if these variations are modeled using a statistical distribution $f_{\bar{E}}(\bar{E})$, then the global distribution of the system under consideration will be

$$
P(E)=\int_{0}^{+\infty} f_{E}(E \mid \bar{E}) f_{\bar{E}}(\bar{E}) d \bar{E},
$$

where $f_{E}(x \mid \bar{E})$ is the Boltzmann distribution with mean $\bar{E}$. Given a model $f_{\bar{E}}(\bar{E})$ of fluctuations of $\bar{E}$ and the relationship (3) linking $\beta$ and $\bar{E}$, we readily obtain the distribution for $\beta$ :

$$
f_{\beta}(\beta)=f_{\bar{E}}\left(\frac{-d \Phi(\beta)}{d \beta}\right)\left|\frac{d^{2} \Phi(\beta)}{d \beta^{2}}\right| .
$$

Then, the system distribution can also be written

$$
P(E)=\int_{0}^{+\infty} f_{E}(E \mid \beta) f_{\beta}(\beta) d \beta .
$$

Of course this is similar to the 'Superstatistics' of Beck and Cohen [3], but with the supplementary ingredient of the relationship between the intensive parameter $\beta$ and its dual variable $\bar{E}$, so that a model of fluctuations can be naturally introduced for $\bar{E}$. At this point, it can also be noted that the dimension of $\beta$ is the inverse of an energy $E$.

\section{Illustration in the canonical case}

Let us now illustrate this point of view with a very simple example: the probability distributions of velocity and kinetic energy for a macroscopic body consisting of a large number of atoms. In the Gibbs distribution, the energy $E(p, q)$ can be decomposed in the sum of the kinetic and potential energy $E(p, q)=K(p)+U(q)$, so that $e^{-\beta E(p, q)} d p d q=$ $e^{-\beta K(p)} e^{-\beta U(q)} d p d q$, with $p$ the momenta and $q$ the coordinates. Then the probability for the momenta reduces to $A e^{-\beta K(p)} d p$. We know that for an atom of mass $m$ the kinetic energy is $K(p)=\left(p_{x}^{2}+p_{y}^{2}+p_{z}^{2}\right) / 2 m$, where $p_{x}, p_{y}, p_{z}$ are the coordinates of the momentum, and one obtains the classical Maxwellian distribution. Expressing the distribution in terms of the velocities, with $p=m v$ and $v^{2}=v_{x}^{2}+v_{y}^{2}+v_{z}^{2}$, we have

$$
f_{V}(v \mid \beta)=4 \pi\left(\frac{\beta m}{2 \pi}\right)^{3 / 2} v^{2} e^{-\beta m v^{2} / 2}
$$


for the magnitude of the velocity, and

$$
f_{E}(E \mid \beta)=\frac{2}{\sqrt{\pi}} \beta^{\frac{3}{2}} \sqrt{E} e^{-\beta E}
$$

for the magnitude of the kinetic energy, which is a $\chi^{2}$ distribution with three degrees of freedom. If the motion was restricted to a single axis, we would have a single degree of freedom and an exponent $1 / 2$ for $\beta$ instead of $3 / 2$ in the previous relations. The partition function is given by

$$
Z(\beta)=\int_{0}^{+\infty} e^{-\beta K(p)} d p=\left[\frac{\pi}{2 \beta m}\right]^{\frac{3}{2}},
$$

and we immediately obtain

$$
\bar{E}=-\frac{d}{d \beta} \log Z(\beta)=\frac{3}{2 \beta} .
$$

Therefore, in the canonical case, the mean value of the kinetic energy is simply proportional to the inverse of the parameter $\beta$. Fluctuations of this mean value $\bar{E}$ can be modeled through a distribution $f_{\bar{E}}$.

\section{The example of K-distributions}

It is well known, e.g. [2,10], that Gamma fluctuations of the 'inverse temperature' $\beta$ leads to a Tsallis distribution. Because of the relationship (10), this corresponds to an inverse-Gamma distribution for $\bar{E}$. Another reasonable model for fluctuations of the mean energy $\bar{E}$ is a Gamma distribution since it proves highly flexible for modeling the distribution of positive variables. It also appears as the distribution of the sum of squared Gaussian variables.

\subsection{Derivation}

Therefore, according to (10), $\beta$ is distributed according to the inverse-Gamma distribution: $f_{\beta}(\beta)=\frac{b^{a}}{\Gamma(a)} \beta^{-a-1} e^{-\frac{b}{\beta}}$. Then, $P(E)$ is given by

$$
P(E)=\frac{2}{\sqrt{\pi}} \frac{b^{a}}{\Gamma(a)} \int_{0}^{+\infty} \beta^{\frac{3}{2}} \sqrt{E} e^{-\beta E} \beta^{-a-1} e^{-\frac{b}{\beta}} d \beta .
$$

Formula [3.471.9] in [11] enables to perform the integration with respect to $\beta$. This formula reads $\int_{0}^{+\infty} x^{\nu-1} e^{-\frac{\beta}{x}-\gamma x} d x=$ $2\left(\frac{\beta}{\gamma}\right)^{\frac{\nu}{2}} K_{\nu}(2 \sqrt{\beta \gamma})$ with $\beta, \gamma>0$, and where $K_{\nu}$ is a modified Bessel function the of second kind of order $\nu$. This leads to

$$
P(E)=\frac{2}{E} \frac{1}{\Gamma(a) \Gamma(3 / 2)}(b E)^{\frac{3 / 2+a}{2}} K_{-\frac{3}{2}+a}(2 \sqrt{b E}) .
$$

As far as the velocity is concerned, the variable change $E=\frac{1}{2} m v^{2}$ gives

$$
P_{V}(v)=\frac{4}{\Gamma(3 / 2) \Gamma(a)} v^{a+\frac{1}{2}} c^{a+\frac{3}{2}} K_{a-\frac{3}{2}}(2 c v)
$$

with $c=\sqrt{b m / 2}$.

\subsection{Main properties and characterizations}

These distributions are known as K-distributions. They are extensively used for modeling the statistics of interferences of radiowaves, radar clutter, optical scintillation, ultrasound scattering, etc [12,13,14,15,16,17,18,19]. Interestingly, a recent application of inverse-chi-square superstatistics in medical statistics has been proposed [20] with excellent agreement with real data. The shape of distribution (12) and its evolution when the parameter $a$ varies is given in Fig. 1. This distribution is derived in radar imaging as the mixture of a Rayleigh and Gamma distribution [18] and competes with log-normal e.g. [15] or generalized Pareto (Tsallis) distributions e.g. [17] for fitting observed data. It is worth mentioning that the estimation of the parameters of this distribution is well documented, e.g. $[14,16,18]$. 


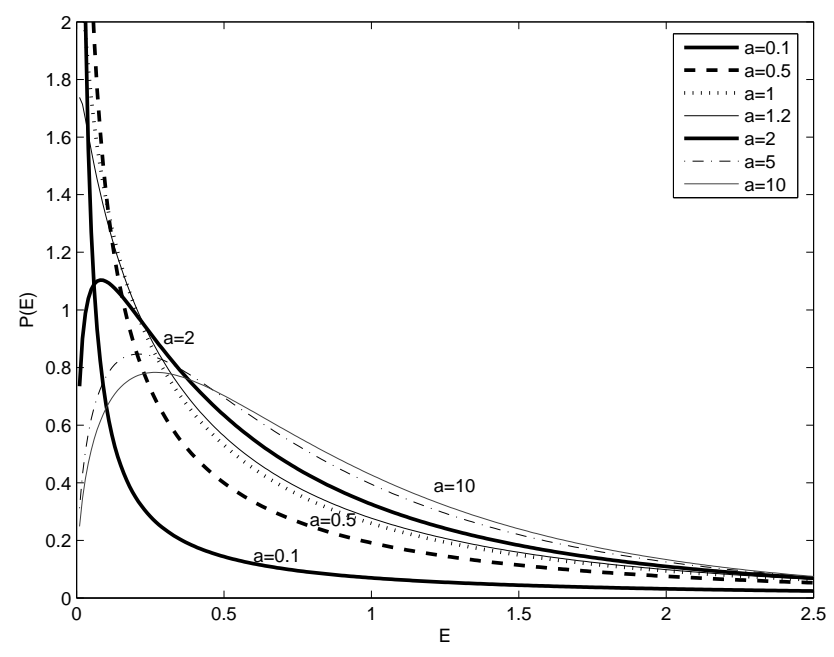

Fig. 1. Examples of K-distributions (12) with $a=0.1$ to $a=10$, and with $b=3 a / 2$ chosen so that the expectation of all distributions is equal to 1 .

The case of general mixture of Gamma distributions was considered in [21] where is given a formula similar to (12). A $\mathrm{K}$-distribution was also obtained in [4] in the limit case of a ratio of two Gamma distributions (that is a F-distribution) for describing fluctuations of $\beta$. A model of inverse chi-square for $\beta$ was mentioned and its asymptotics predicted in [22]. These asymptotics are easy to observe: formula [9.7.2] in [23] gives the limit form for the Bessel $\mathrm{K}$ function for large arguments: $K_{\nu}(z) \approx \sqrt{\frac{\pi}{2 z}} e^{-z}$. Consequently, $P(E)$ behaves as

$$
P(E) \sim \frac{2}{\Gamma(a)}(b E)^{\frac{a-1}{2}} e^{-2 \sqrt{b E}}
$$

and presents an exponential tail. The survival function $\bar{F}(x)=\operatorname{Pr}(X \geq x)$ then has the limit form $\bar{F}(x)=$ $\frac{1}{\Gamma(a) b}\left(\frac{1}{2}\right)^{a-1} \Gamma(a+1,2 \sqrt{b x})$, where $\Gamma(a+1,2 \sqrt{b x})$ is the upper incomplete Gamma function. It behaves as $(2 \sqrt{b x})^{a} e^{-2 \sqrt{b x}}$ for large values of $x$ and $\lim _{x \rightarrow+\infty} e^{\lambda x} \bar{F}(x)=+\infty$ for all positive $\lambda$, which means that the distribution is heavytailed.

For small values of the argument, [23] again gives a limiting form, formula [9.6.9]: $K_{\nu}(z) \approx \frac{1}{2} \Gamma(\nu)\left(\frac{1}{2} z\right)^{-\nu}$ for $\nu>0$. For $a>3 / 2$ we then have

$$
P(E) \sim \frac{\Gamma\left(a-\frac{3}{2}\right)}{\Gamma(a) \Gamma(3 / 2)} b^{\frac{3}{2}} \sqrt{E} .
$$

The case $a<3 / 2$ follows using the fact that $K_{-\nu}(z)=K_{\nu}(z)$ :

$$
P(E) \sim \frac{\Gamma\left(\frac{3}{2}-a\right)}{\Gamma(a) \Gamma(3 / 2)} b^{a} E^{a-1} .
$$

This last expression shows that the K-distribution presents an integrable singularity at the origin for $a<1$, and explains the behavior shown in Fig. 1. In such a case, there is a preferential weighting of small values interrupted by some rare large events, an interesting feature encountered in many experiments. A typical realization obtained with $a=0.5$ is reported in Fig. 2 .

As a final comment, let us recall that the distribution of the ratio of a Gaussian variable and a Gamma variable is a Tsallis distribution. Along the same line, a stochastic model of K-distributed variates can be a simple multiplicative model. Indeed, if $z$ is the product of two Gamma variables $x$ and $y$, the distribution of $z$ is $p_{Z}(z)=\int p_{X}(x) p_{Y}(z / x) d x / x$, which reduces to a $\mathrm{K}$-distribution. 


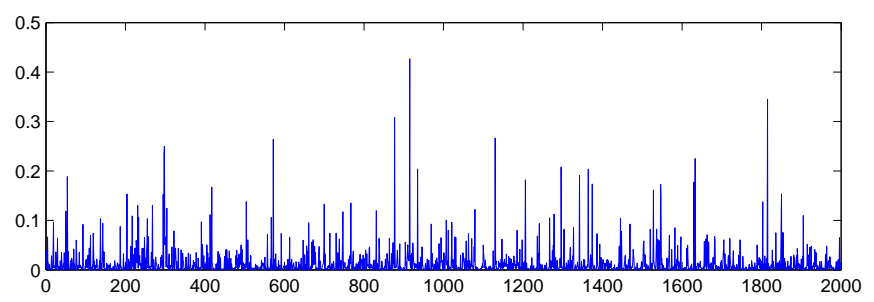

Fig. 2. A typical realization of a K-distribution with $a=0.5$.

\section{Conclusion}

This Letter presents two contributions. First, we have highlighted the fact that within a maximum entropy setting, the $\beta$ parameter and observable are conjugated, and linked by relation (3). Therefore, their fluctuations are also linked. This gives an alternate viewpoint to 'Superstatistics', since the model of fluctuations can be introduced for the observable rather than for the intensive parameter.

As an illustration and second contribution, we worked out the natural example of a Gamma distribution, and exhibited the system distribution in the form of a K-distribution. We underlined that this interesting distribution is highly relevant in some physical applications, and have emphasized some of its properties.

\section{References}

[1] N. Johnson, S. Kotz, A. Kemp, Univariate Discrete Distributions, 2nd Edition, John Wiley, New York, 1992.

[2] G. Wilk, Z. Wlodarczyk, Interpretation of the nonextensitivity parameter $q$ in some applications of Tsallis statistics and Lévy distributions, Phys.Rev.Lett. 84 (2000) 2770.

[3] C. Beck, E. Cohen, Superstatistics, Physica A 322 (2003) 267-275.

[4] F. Sattin, L. Salasnich, Multiparameter generalization of nonextensive statistical mechanics, Phys. Rev. E 65 (2002) 035106.

[5] A. G. Bashkirov, On maximum entropy principle, superstatistics, power-law distribution and Rényi parameter, Physica A 340 (2004) 153-162.

[6] S. M. D. Queirós, On the emergence of a generalised Gamma distribution. Application to traded volume in financial markets, Europhys. Lett. 71 (3) (2005) pp. 339-345.

[7] T. Yamano, Thermodynamical and informational structure of superstatistics, Progress of Theoretical Physics Supplement 162 (2006) 87-96.

[8] G. Wilk, Z. Wlodarczyk, Fluctuations, correlations and the nonextensivity, Physica A: Statistical and Theoretical Physics 376 (2007) $279-288$.

[9] S. Abe, C. Beck, E. G. D. Cohen, Superstatistics, thermodynamics, and fluctuations, Phys. Rev. E 76 (2007) $031102-5$.

[10] B. A. Maguire, E. S. Pearson, H. A. Wynn, The time intervals between industrial accidents, Biometrika 39 (1952) $168-180$.

[11] I. Gradshteyn, I. Ryzhik, Table of Integrals, Series and Products, Academic Press, 1965.

[12] E. Jakeman, P. N. Pusey, Significance of K distributions in scattering experiments, Physical Review Letters 40 (9) (1978) 546-550.

[13] E. Jakeman, On the statistics of K-distributed noise, Journal of Physics A 13 (1980) 31-48.

[14] R. S. Raghavan, A method for estimating parameters of K-distributed clutter, IEEE Transactions on Aerospace and Electronic Systems 27 (2) (1991) 238-246.

[15] A. Abdi, M. Kaveh, K distribution: an appropriate substitute for Rayleigh-lognormal distribution in fading-shadowing wireless channels, Electronics Letters 34 (1998) 851-852.

[16] D. R. Iskander, A. M. Zoubir, B. Boashash, A method for estimating the parameters of the K-distribution, IEEE Trans. Signal Processing 47 (1999) 1147-1151.

[17] B. LaCour, Statistical characterization of active sonar reverberation using extreme value theory, IEEE Journal of Oceanic Engineering 29 (2004) 310-316.

[18] K. D. Ward, R. J. A. Tough, S. Watts, Sea Clutter: Scattering, the K Distribution And Radar Performance, Institution for Engineering and Technology, 2006.

[19] L. Weng, J. M. Reid, P. M. Shankar, K. Soetanto, Ultrasound speckle analysis based on the K-distribution, The Journal of the Acoustical Society of America 89 (1991) 2992-2995.

[20] L. L. Chen, C. Beck, A superstatistical model of metastasis and cancer survival, Physica A (in press) (2008). URL http: / / arxiv.org/abs/0711.4687

[21] S. K. Bhattacharya, A modified Bessel function model in life testing, Metrika 11 (1966) 133-144.

[22] H. Touchette, C. Beck, Asymptotics of superstatistics, Phys. Rev. E 71 (2005) 016131-6.

[23] M. Abramowitz, I. Stegun, Handbook of Mathematical Functions: with Formulas, Graphs, and Mathematical Tables, Dover Publications, 1965. 